\title{
Evaluating Efficacy of Mandibular Advancement Device in Patients With Essential Hypertension and Obstructive Sleep Apnea
}

\author{
Manan Shah, MDS; Somil Mathur, MDS; Snehal Upadhyay, MDS; Rakesh Makwana, MDS
}

\begin{abstract}
Department of Prosthodontics, Crown and Bridgework and Oral Implantology, Faculty of Dental Science, Dharmsinh Desai University, Nadiad, Gujarat, India
\end{abstract}

\begin{abstract}
Study Objectives: Obstructive sleep apnea (OSA) has a close association with hypertension and often leads to uncontrolled hypertension. One treatment modality for mild to moderate OSA is the mandibular advancement device (MAD). The goal of the current study was to evaluate the effect of the MAD on blood pressure and sleep apnea severity.

Methods: A total of 25 participants, screened for OSA using the STOP-Bang Questionnaire, and underwent a level 3 home sleep test. Based on apnea-hypopnea index (AHI) calculated from a level 3 home sleep test, selected participants with mild to moderate OSA underwent 24-hour ambulatory blood pressure monitoring. The monoblock type of custom-made MAD was fabricated for each of 21 selected participants. After 1 month of MAD therapy, a level 3 home sleep apnea test was performed again with the MAD in position and 24-hour ambulatory blood pressure monitoring were repeated for comparison with both parameters before MAD therapy.

Results: MAD therapy resulted in significant reduction of systolic and diastolic blood pressure $(\mathrm{p}<0.05)$ and simultaneous reduction in AHI was also reported $(P<0.05)$.

Conclusions: MAD therapy is associated with significant reduction in AHI and ambulatory blood pressure in subjects with essential hypertension and OSA.

Keywords: essential hypertension, level 3 home sleep apnea test, mandibular advancement device, obstructive sleep apnea Citation: Shah M, Mathur S, Upadhyaya S, Makwana R. Evaluating efficacy of mandibular advancement device in patients with essential hypertension and obstructive sleep apnea. J Dent Sleep Med. 2020;7(4)
\end{abstract}

\section{INTRODUCTION}

Obstructive sleep apnea (OSA) is a common disease that usually occurs because of narrowing in the size of the upper airway during sleep and is believed to be a leading cause of transient asphyxia. This event leads to intermittent hypoxemia, sleep disturbances, daytime somnolence, brain arousals, and poor quality of life. As a result of the aforementioned events, the cardiovascular system may already be adversely affected by metabolic and neurohormonal disturbances. ${ }^{1}$ Epidemiologic data suggest that there is a strong association between OSA and systemic hypertension and that this association affects cardiovascular outcomes. The upper airway muscular activity is inhibited by an acute increase in blood pressure. This occurrence, together with volume overburden and its relocation to the upper body during rest, which can be experienced by individuals with hypertension, leads to pharyngeal edema and may clarify the connection among hypertension and OSA. ${ }^{2}$

It is reported that continuous positive airway pressure therapy for OSA improves blood pressure of patients with hypertension. ${ }^{3}$ A few studies have depicted the antihypertensive effect resulting from mandibular advancement device (MAD) therapy on mild to moderate OSA. ${ }^{4-6}$

Based on the apnea-hypopnea index (AHI) calculated from polysomnographic analysis, OSA can be categorized as mild ( $\mathrm{AHI}=5$ to 15 events/hour), moderate (AHI $=15$ to 30 events/hour), and severe $(\mathrm{AHI}=$ more than 30 events/hour). ${ }^{7}$ Many studies have revealed that use of MAD therapy is an effective treatment to reduce severity of mild to moderate OSA by $50 \%{ }^{8-10}$ MADs relieve snoring and sleep apnea by widening or opening the airway through anterior positioning of the mandible during sleep. ${ }^{11,12}$

Many subjective and objective means of diagnosis of OSA have been reported to date. The STOP-Bang questionnaire includes both subjective and objective parameters and is used for screening of patients with significant risk of OSA. ${ }^{13}$

Subjective parameters are used to assess the history of snoring, observed apnea, and tiredness whereas objective parameters includes body mass index, blood pressure, neck circumference, age, and sex of the participants. The Epworth Sleepiness Scale (ESS) is a subjective questionnaire to assess daytime sleepiness in participants with OSA. ${ }^{14}$ Polysomnography is the most common and gold standard objective analysis for diagnosis and monitoring of OSA that is noninvasive and does not require any radiation. It is possible to obtain reports of different parameters such as electrocardiography, electroencephalography, electromyography, electrooculography, respiratory events, oxygen saturation, body 
movements, and snoring loudness, depending on level of polysomnography. Polysomnography can be done in the laboratory as well as at home. Polysomnography performed at home is also called a level 3 home sleep apnea test, which includes electrocardiography or heart rate monitoring, oxygen saturation, and at least two channels of respiratory movement. ${ }^{7,15,16}$

The current study was designed to evaluate the effect of MAD therapy on OSA severity in patients with essential hypertension. The null hypothesis was that the MAD would not have any effect on blood pressure or subjective and objective symptoms of the participants with OSA.

\section{MATERIALS AND METHODS}

The current prospective cohort study was conducted in the Department of Prosthodontics, Crown and Bridgework and Oral Implantology, Faculty of Dental Science, Dharmsinh Desai University, Nadiad. Twentyfive participants were screened for OSA with the help of a STOP- Bang Questionnaire. All participants underwent a level 3 home sleep test (ApneaLink Air, Martinsried, Germany) which uses portable monitors that allow sleep studies to be done at home (Figure 1). Participants who were not willing to undergo nasal continuous positive airway pressure therapy were selected for the study, and written informed consent was obtained from each participant. All participants filled out a demographic questionnaire which included age, sex, alcohol ingestion, smoking habits, and history of hypertension. Neck circumference (measured just below the prominence of the thyroid cartilage), body mass index and Mallampati scores were also recorded. Daytime sleepiness was assessed using the ESS. Participants with inadequate nasal airflow capacity, less than $7 \mathrm{~mm}$ protrusive jaw movement from maximum intercuspation, temporomandibular joint disorder, fewer than three healthy teeth per quadrant, severe periodontitis, and high caries index were excluded from the study. ${ }^{18}$

Figure 1. Polysomnographic Armamentarium

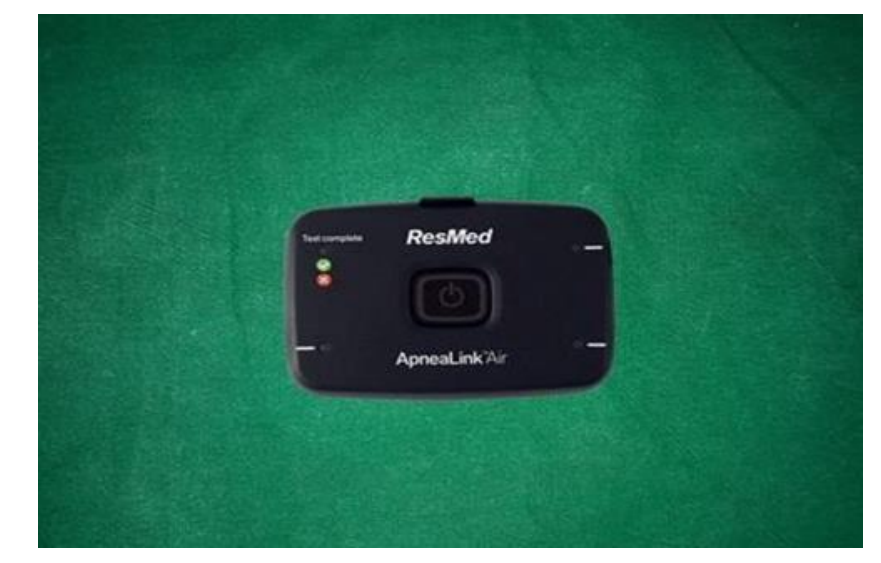

Based on AHI calculated from the level 3 home sleep test, three participants with severe OSA were excluded from the study. Following the level 3 home sleep apnea test, 24-hour systolic and diastolic ambulatory blood pressure was measured using an electric automated manometer (Krishkare, India) (Figure 2). Based on 2017 American Heart Association classification of hypertension, one participant with systolic blood pressure less than $140 \mathrm{~mm}$ $\mathrm{Hg}$ and diastolic blood pressure less than $90 \mathrm{~mm} \mathrm{Hg}$ was excluded from the study. ${ }^{17}$

\section{Figure 2. Automated Manometer}

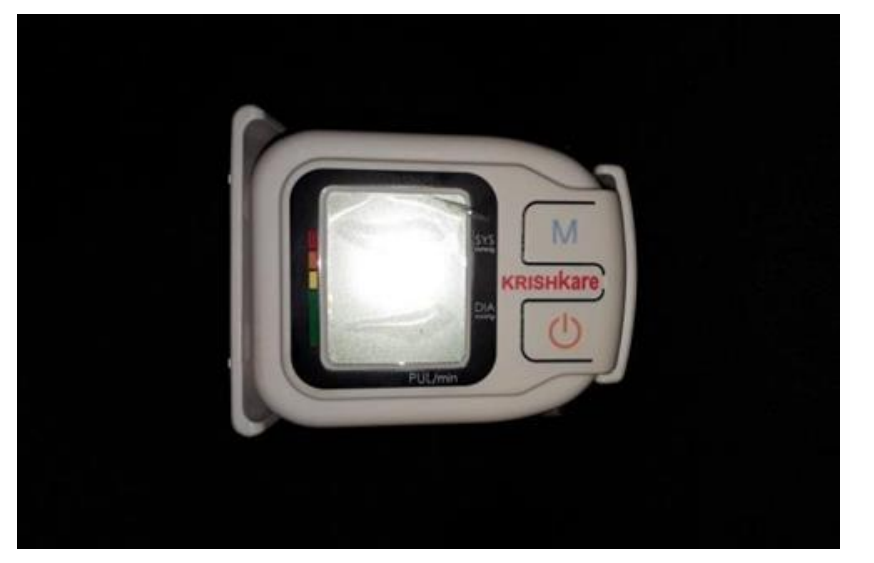

Maxillary and mandibular diagnostic impressions were made with irreversible hydrocolloid impression material (Imprint, DPI, India) and casts were poured using type 3 dental stone (Kalabhai, Mumbai, India) (Figure 3). In maximum intercuspation (P1), two vertical lines were marked on casts: one from the buccal surface of the first premolar and molar to the buccal surface of the maxillary premolar and molar, respectively (Figure 3). A protrusive interocclusal record was made using aluminum-reinforced wax (Morsa, Bavaria, Germany) at $75 \%$ of the maximum mandibular protrusion (P2) (Figure 4). Seventy-five percent of maximum mandibular protrusion (P2) was recorded by measuring the distance between the labial surface of the maxillary incisor to the labial surface of the mandibular incisor with a vernier caliper. This record was transferred to casts and $75 \%$ of maximum mandibular

Figure 3. Diagnostic Casts and Marking

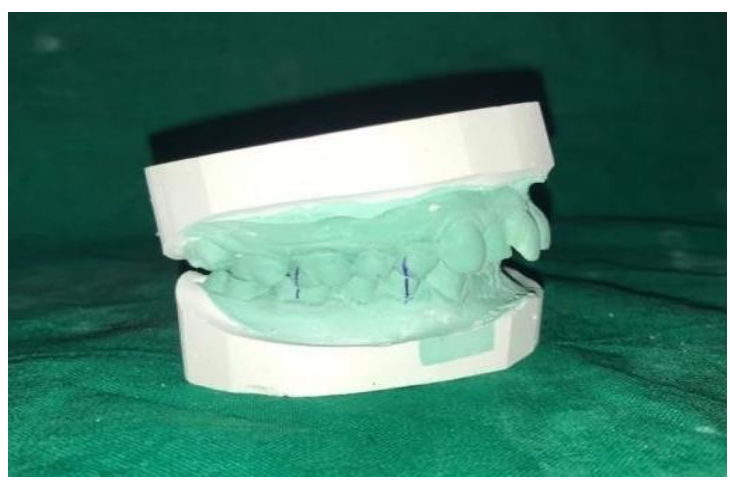




\section{Figure 4. Interocclusal Record}

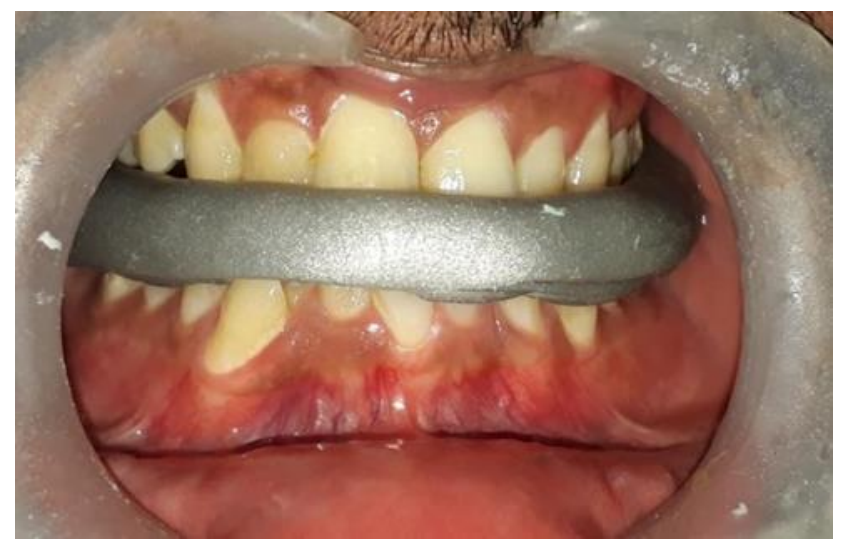

protrusion was verified by the distance between the two previously drawn vertical lines. Both casts were articulated on a mean value articulator using the interocclusal record. Wax for the MAD was made using modeling wax (Y-Dent, India) and processing was done using heat-polymerized acrylic resin (Trevlon, DENTSPLY, India) as per manufacturer instructions (Figures 5 and 6). Minor adjustments were carried out to eliminate processing errors, and verification of $75 \%$ of maximum mandibular protrusion was done. Following delivery of the MAD, participants were recalled after 24 hours for postinsertion adjustments. After 1 month of MAD therapy, all participants were again subjected to a level 3 home sleep apnea test with the MAD in position. Twenty-four-hour ambulatory blood pressure analysis and assessment of daytime sleepiness with ESS were performed. All the data were plotted and compared statistically using paired $t$ test.

Figure 5. Mandibular Advancement Device

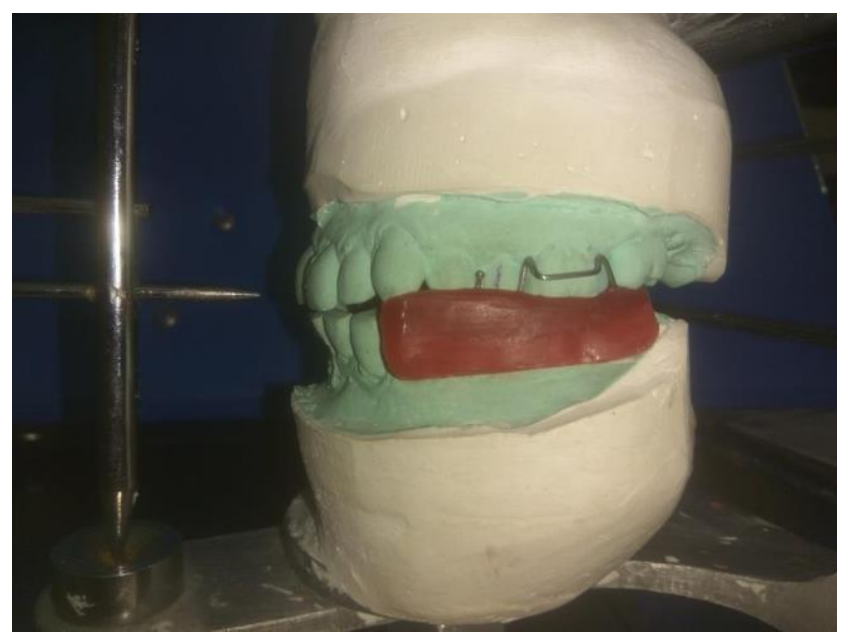

\section{RESULTS}

Demographic characteristics of patients with OSA are outlined in Table 1.
Figure 6. Mandibular Advancement Device

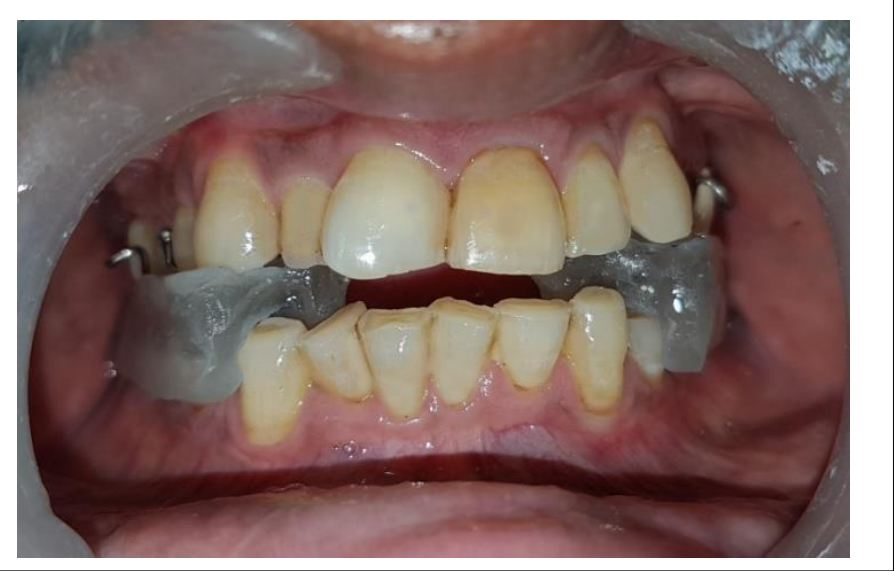

\section{Subjective assessment}

The ESS after MAD therapy $(10.15 \pm 2.06)$ was significantly low when compared to ESS before MAD therapy $(\mathrm{ESS}=15.68 \pm 2.80)(P<0.05)$ Subjective assessment is outlined in Table 2.

\section{Objective assessment}

AHI $(27.25 \pm 16.67$ events per hour $)$ was significantly reduced after MAD therapy in comparison with AHI before MAD therapy (46.66 \pm 15.02 events per hour) (Figure-7). Twenty-four-hour ambulatory mean systolic blood pressure $(140.28 \pm 15.8 \mathrm{mmHg})$ showed significant reduction following MAD therapy $(130.24 \pm 14.1 \mathrm{mmHg})$. Similarly, 24-hour ambulatory mean diastolic blood pressure was also reduced significantly, from $89.64 \pm 10.4$ $\mathrm{mmHg}$ to $85.29 \pm 11 \mathrm{mmHg}$ (Figures 8 and 9).

\section{DISCUSSION}

The goal of the current study was to evaluate the effect of MAD therapy in patients with essential hypertension and OSA. Use of the MAD reduces sleep apnea severity to half and is most effective in mild to moderate cases. The MAD dilates or opens the airway by repositioning the mandible and tongue during sleep, which is helpful in relieving snoring and OSA. OSA is closely associated with high blood pressure. ${ }^{19,20}$ This research was intended to determine whether the use of the MAD in affected individuals had an influence on blood pressure values. The pathogenesis of hypertension in OSA is affected by various factors, including load of non-positive intrathoracic pressure as a result of upper airway occlusion, recurrent arousals, reduction in stretch receptor stimulation of the lung, and temporary hypoxemia. ${ }^{21}$ In addition, multifaceted relationships with the boost of sympathetic nervous activity, inflammation, active enzyme, oxidative stress, and endothelial dysfunction are responsible for an increase in and variation of blood pressure. ${ }^{22,23}$ The antihypertensive effect of MAD therapy is not only because of the 
Figure 7. Apnoea-Hypopnoea index (Before and after MAD therapy)

\section{Apnoea- Hypopnoea Index}

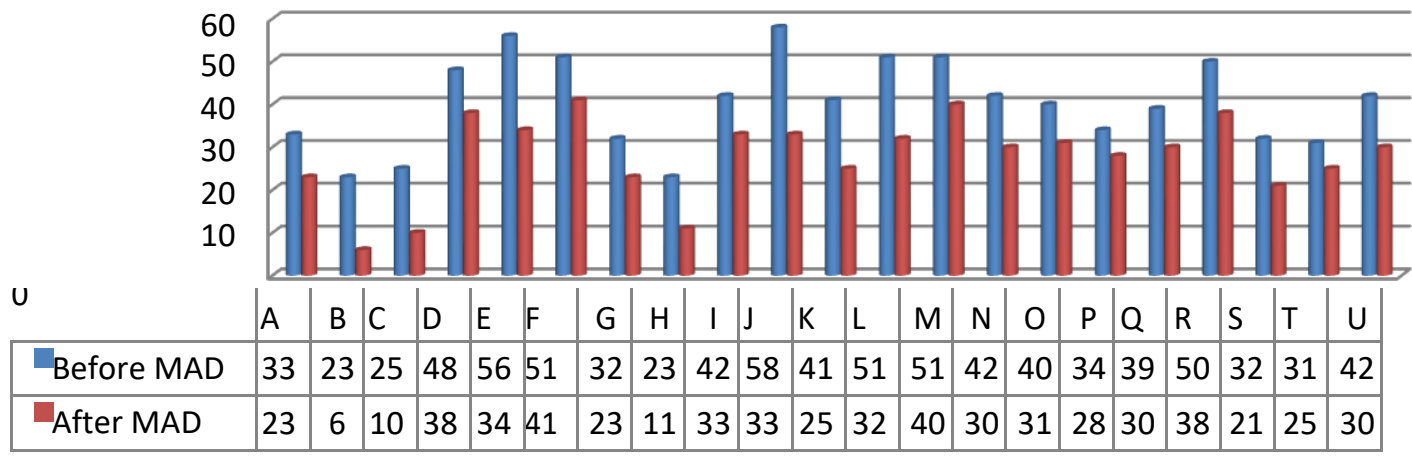

Figure 8. Systolic blood pressure (Before and after MAD therapy)

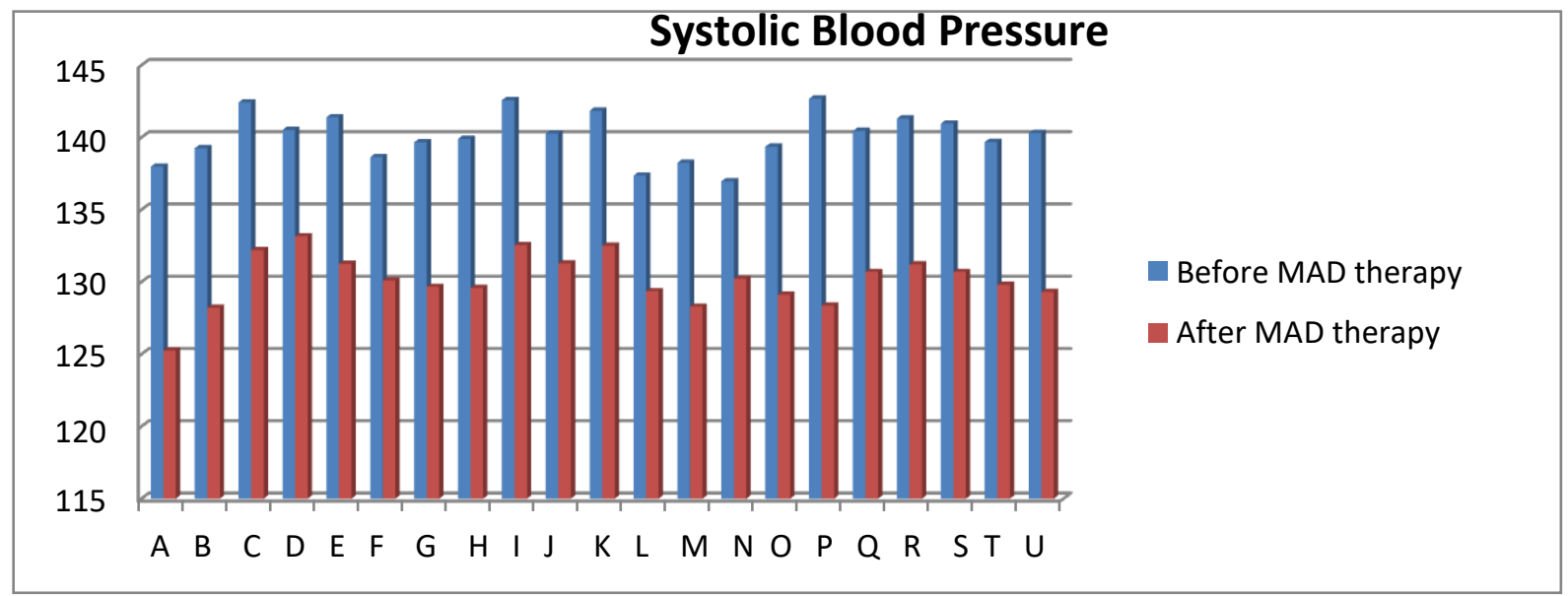

Figure 9. Diastolic blood pressure (Before and after MAD therapy)

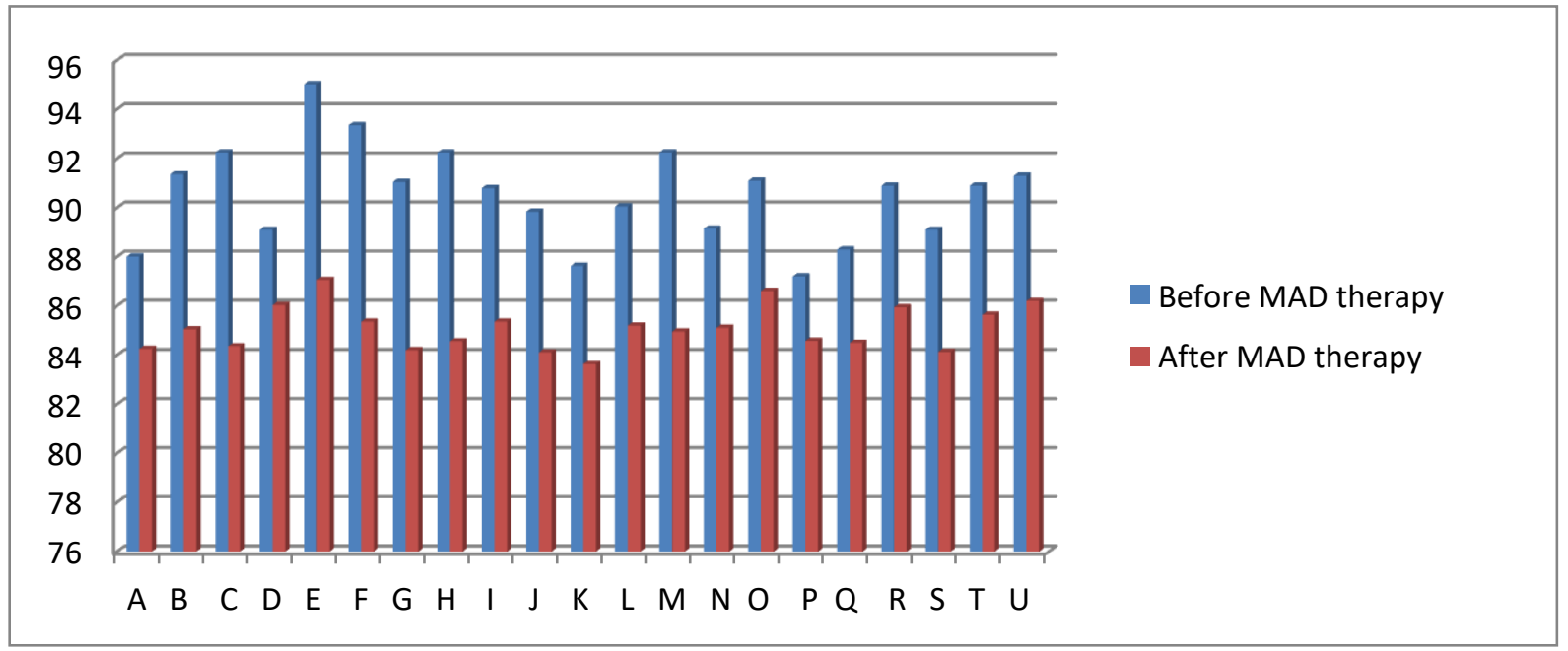


Table 1. Demographic characteristic (mean \pm standard deviation); $(n=21)$ of patients with obstructive sleep apnea.

\begin{tabular}{|c|c|}
\hline Characteristics & Statistics \\
\hline Gender : Male & $15(71.42 \%)$ \\
\hline Female & $6(28.57 \%)$ \\
\hline Age (Years) & $50.04 \pm 10.05(27-65)$ \\
\hline Height $(\mathrm{cm})$ & $160.64 \pm 10.16(149-170)$ \\
\hline Weight (kg) & $75.05 \pm 11.43(60-90)$ \\
\hline Smoking: Yes & $15(71.42 \%)$ \\
\hline No & $6(28.57 \%)$ \\
\hline Diabetes Mellitus: Yes & $11(52.38 \%)$ \\
\hline No & $10(47.61 \%)$ \\
\hline Hypertension: Yes & $21(100 \%)$ \\
\hline No & $0(0.0 \%)$ \\
\hline Snoring: Yes & $21(100 \%)$ \\
\hline No & $0(0.0 \%)$ \\
\hline Mallampati Score: 1 & 0 \\
\hline 2 & 15 \\
\hline 3 & 5 \\
\hline 4 & 1 \\
\hline Neck Circumference (inch) & $16.05 \pm 0.81(15-18)$ \\
\hline Apnoea Hyponea Index (AHI) (events/hour) & $27.25 \pm 16.67(6-56)$ \\
\hline
\end{tabular}

enhancement of negative intrathoracic pressure responsible for an effect on hemodynamics, but also because of improvement of arousal reaction by suppression of sympathetic activity. ${ }^{24,25}$ Significant reduction in blood pressure values in known participants was observed after 1 month of MAD therapy. MADs have the ability to alter dimensions of the upper airway by carrying out an anterior and inferior movement of the jaw that facilitates an increase in the dimension of the airway in the pharyngeal region. ${ }^{26}$ As a result of this movement, stabilization of the jaw and the hyoid bone takes place, which opens the airway and prevents the posterorotation of these structures during the decubitus. It shows consequences for all the pharyngeal segments, though its main effect appears in the velopharyngeal area. Rigidity and swelling occurs between the anterior and posterior pharyngeal pillars. Ventral 
Table 2. Summary of subjective assessment.

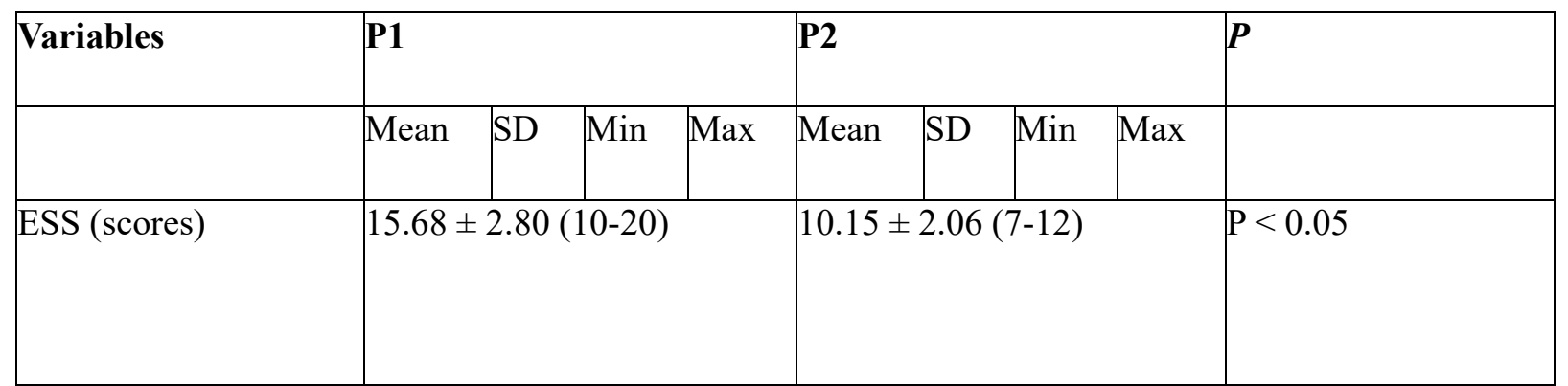

ESS, Epworth Sleepiness Scale; P1, maximum intercuspation; P2, 75\% of maximum protrusion; $\mathrm{SD}$, standard deviation.

displacement of the soft palate, and thereby an increase in the caliber of the lateral walls of the velopharyngeal area, are the main factors for reduction in snoring. Functional MADs enable the hyoid bone to occupy a more forward position. As a result of this, a new position of balance of the suprahyoid musculature is created, which in turn increases the permeability and volume of the upper airway. Because of attachment of palatoglossi muscles, lateral relocation of the pharyngeal fat pads from the airway occurs and the base muscles of the tongue move anteriorly, which reduces pharyngeal collapsibility in lateral dimension. ${ }^{27}$ In the current study, after 1 month of MAD usage the AHI was significantly reduced. These values are in correlation with the other studies..$^{28,29}$ The ESS is a scale intended to measure daytime sleepiness. Tendency to doze off or fall asleep unintentionally during the day is termed excessive daytime sleepiness. Induction of sleepiness/fatigue retards performance capability due to increased phases of non-responding or delayed response time, increased reaction times, slow information processing, reduced vigilance, reduced precision about short-term memory, and accelerated decrements in performance with time on task. ${ }^{30}$ This leads to an increase in human error and risk for accidents. Cumulative evidence indicates that the probability of motor vehicle crashes and sleepiness are directly proportional. The current study used the ESS to subjectively assess participants' daytime sleepiness for this type of treatment and it was found that the results were in agreement with other similar studies.6,21,22 Thus, the null hypothesis that the MAD would not have any effect on subjective and objective symptoms of the participants was rejected. Because the current study has limited sample size and shows short-term results, further studies with large sample size and long-term follow-up would be more helpful in understanding the complex relationship between OSA and essential hypertension.

\section{CONCLUSION}

On the basis of the findings and within the limitations of the current study, it can be concluded that use of MAD

therapy improves objective and subjective symptoms at $75 \%$ of maximum intercuspation position. MAD usage in patients with OSA and hypertension can lead to reduction of blood pressure and AHI. This reduction may attribute to improvement of sleep quality and overall health of an individual.

\section{ABBREVIATIONS}

AHI, apnea-hypopnea index

ESS, Epworth Sleepiness Scale MAD, mandibular advancement device

OSA, obstructive sleep apnea

\section{REFERENCES}

1. Cappuccio FP, Miller MA. Sleep and cardio-metabolic disease. Curr Cardiol Rep. 2017;19(11):110. doi: 10.1007/s11886-017-0916-0.

2. Mansukhani MP, Covassin N, Somers VK. Apneic sleep, insufficient sleep, and hypertension. Hypertension. 2019;73(4):744-756. doi: 10.1161/HYPERTENSIONAHA.118.11780.

3. Bazzano LA, Khan Z, Reynolds K, He J. Effect of nocturnal nasal continuous positive airway pressure on blood pressure in obstructive sleep apnea. Hypertension. 2007;50(2):417-423. DOI:10.1161/HYPERTENSIONAHA.106.085175

4. Yoshida K. Effect on blood pressure of oral appliance therapy for sleep apnea syndrome. Int J Prosthodont. 2006;19(1):61-66.

5. Rietz H, Franklin KA, Carlberg B, Sahlin C, Marklund M. Nocturnal blood pressure is reduced by a mandibular advancement device for sleep apnea in women: Findings from secondary analyses of a randomized trial. J Am Heart Assoc. 2018;7(13). pii: e008642. doi: 10.1161/JAHA.118.008642.

6. Bratton DJ, Gais1 T, Wons AM, Kohler M. CPAP vs mandibular advancement devices and blood pressure in patients with obstructive sleep apnea: A systematic review and meta-analysis. JAMA. 2015;314(21):2280-2293. doi: 10.1001/jama.2015.16303.

7. Kapur VK, Auckley DH, Chowdhuri S, et al. Clinical Practice 
Guideline for Diagnostic Testing for Adult Obstructive Sleep Apnea: An American Academy of Sleep Medicine Clinical Practice Guideline. $\quad J$ Clin Sleep Med. 2017;13(3):479-504. doi: 10.5664/jcsm.6506.

Schmidt-Nowara WW, Meade TE, Hays MB. Treatment of snoring and obstructive sleep apnea with a dental orthosis. Chest. 1991;99(6):1378-1385.

Clark GT, Arand D, Chung E, Tong D. Effect of anterior mandibular positioning on obstructive sleep apnea. Am Rev Respir Dis. 1993;147(3):624-629.

10.

Demko BG. The evolution of oral appliance therapy for snoring and sleep apnea: Where did we come from, where are we, and where are we going?. Sleep Med Clin. 2018; 13(4):467-487. doi: 10.1016/j.jsmc.2018.07.001.

11. Rashmikant US, Chand P, Singh SV, et al. Cephalometric evaluation of mandibular advancement at different horizontal jaw positions in obstructive sleep apnoea patients: a pilot study. Aust Dent J. 2013;58(3):293-300.

Okuno K, Pliska BT, Hamoda M, Lowe AA, Almeida FR. Prediction of oral appliance treatment outcomes in obstructive sleep apnea: A systematic review. Sleep Med Rev. 2016;30:25-33. doi: 10.1016/j.smrv.2015.11.007.

13. Chiu HY, Chen PY, Chuang LP. Diagnostic accuracy of the Berlin questionnaire, STOP-BANG, STOP, and Epworth sleepiness scale in detecting obstructive sleep apnea: A bivariate meta-analysis. Sleep Med Rev. 2017;36:57-70. doi: 10.1016/j.smrv.2016.10.004.

14. Johns MW. A new method for measuring daytime sleepiness: the Epworth sleepiness scale. Sleep. 1991;14(6):540-545. DOI:10.1093/sleep/14.6.540

15. Rosen IM, Kirsch DB, Carden KA, et al. Clinical use of a home sleep apnea test: An updated American Academy of Sleep Medicine Position Statement. J Clin Sleep Med. 2018; 15;14(12):2075-2077.

16. El Shayeb M, Topfer L-A, Stafinski T, Pawluk L, Menon D. Diagnostic accuracy of level 3 portable sleep tests versus level 1 polysomnography for sleep-disordered breathing: a systematic review and meta-analysis. CMAJ 2014;186(1):E25-51.

17. Whelton PK, Carey RM, Aronow WS, et al. 2017 ACC/AHA/AAPA/ABC/ACPM/AGS/APhA/ASH/ASPC/NMA/PC

NA Guideline for the Prevention, Detection, Evaluation, and Management of High Blood Pressure in Adults: A Report of the American College of Cardiology/American Heart Association Task Force on Clinical Practice Guidelines. Hypertension. 2018;71(6):e13e115. doi: 10.1161/HYP.0000000000000065.

18. Ramar K, Dort LC, Katz SG, et al. Clinical Practice Guideline for the Treatment of Obstructive Sleep Apnea and Snoring with Oral Appliance Therapy: An Update for 2015. J Clin Sleep Med. 2015;11(7):773-827. doi: 10.5664/jcsm.4858.

19. Cai A, Wang L, Zhou Y. Hypertension and obstructive sleep apnea. Hypertens Res. 2016; 39(6):391-395. doi: 10.1038/hr.2016.11

20. Jafari B. Sleep architecture and blood pressure. Sleep Med Clin. 2017;12(2):161-166. doi: 10.1016/j.jsmc.2017.02.003

21. Iftikhar IH, Hays ER, Iverson MA, Magalang UJ, Maas AK. Effect of oral appliances on blood pressure in obstructive sleep apnea: a systematic review and meta-analysis. $J$ Clin Sleep Med. 2013;9(2):165-174. doi: 10.5664/jcsm.2420.

22. Arnardottir ES, Mackiewicz M, Gislason T, Teff KL, Pack AI. Molecular signatures of obstructive sleep apnea in adults: a review and perspective. Sleep. 2009;32(4):447-470.

23. Kario K. Obstructive sleep apnea syndrome and hypertension: mechanism of the linkage and 24-h blood pressure control. Hypertens Res. 2009;32(7):537-541.

24. Gotsopoulos H, Kelly JJ, Cistulli PA. Oral appliance therapy reduces blood pressure in obstructive sleep apnea: a randomized, controlled trial. Sleep. 2004;27(5):934-941.

25. Sekizuka H, Osada N, Akashi YJ. Effect of oral appliance therapy on blood pressure in Japanese patients with obstructive sleep apnea. Clin Exp Hypertens. 2016;38(4):404-408.

26. Rodríguez-Lozano FJ, Sáez-Yuguero M del R, Linares Tovar E, Bermejo Fenoll A. Sleep apnea and mandibular advancement device. Revision of the literature. Med Oral Patol Oral Cir Bucal. 2008;13(9):E549-554.

27. Aarab G, Lobbezoo F, Hamburger HL, Naeije M. Effects of an oral appliance with different mandibular protrusion positions at a constant vertical dimension on obstructive sleep apnea. Clin Oral Investig. 2010;14(3):339-345.

28. Pitsis AJ, Darendeliler MA, Gotsopoulos H, Petocz P, Cistulli PA. Effect of vertical dimension on efficacy of oral appliance therapy in obstructive sleep apnea. Am $J$ Respir Crit Care Med. 2002;166(6):860-864.

29. Tegelberg A, Walker-Engström M-L, Vestling O, Wilhelmsson B. Two different degrees of mandibular advancement with a dental appliance in treatment of patients with mild to moderate obstructive sleep apnea. Acta Odontol Scand. 2003;61(6):356-362

30. Jaiswal A. Occupational injuries related to sleepiness in Indian traditional industries. Human Biology Review. 2012;21(3):248-267.

\section{SUBMISSION \& CORRESPONDENCE INFORMATION}
Submitted for publication January 22, 2020
Submitted in final revised form May 18, 2020
Accepted for publication March 29, 2020

Address correspondence to: Manan Shah, MDS, shahmanan973@gmail.com

\section{DISCLOSURE STATEMENT}

The authors have no conflicts of interest to disclose. 